\title{
Robust Process Windows for Laser Induced Forward Transfer of Thin Film Metal to Create Interconnects.
}

\author{
G. Oosterhuis ${ }^{1}$, M.P. Giesbers ${ }^{1}$, P.A.J. van Melick ${ }^{1}$, M.B. Hoppenbrouwers ${ }^{1}$, A. M Prenen ${ }^{2}$, A.J. Huis in 't Veld ${ }^{1,3}$ G. Knippels $^{4}$ \\ ${ }^{1}$ TNO, De Rondom 1, 5612 AP Eindhoven, Netherlands \\ ${ }^{2}$ Holst Centre/TNO, Eindhoven, Netherlands \\ ${ }^{3}$ University of Twente, Enschede, Netherlands \\ ${ }^{4}$ ALSI, Beuningen, Netherlands \\ Corresponding author: gerrit.oosterhuis@tno.nl
}

\begin{abstract}
Direct-write technologies can form a low-cost, alternative approach to create electrical interconnects by eliminating mask and etch costs. Also, direct-write is more efficient in creating complex structures as well as for producing small series. However, existing, industrially-mature direct-write technologies typically lack the resolution required for advanced IC packaging applications [1-4]. Laser Induced Forward Transfer (LIFT) is a direct write process which has been proven to be capable of patterning resolutions in the 1-5 $\mu \mathrm{m}$ range [5-8]. Thus far, a lack of deposition control resulting in contamination of the substrate has been a problem. The current paper shows an approach to come to a robust, contamination-free process window for LIFT of pure copper. Thus, we tackled a major roadblock towards the industrial feasibility of LIFT as a full metal direct-write technology that meets the current demands for IC packaging and integration.
\end{abstract}

\section{Introduction}

To be able to write structures that are compatible with advanced packaging demands, a deposit resolution of $1-5 \mu \mathrm{m}$ is required [4]. Laser Induced Forward Transfer (LIFT, Figure 1) has proven to be capable of such resolutions [5-8], hence it is a cost effective alternative for conventional patterned metallization schemes.

TNO, in close cooperation with ALSI, Holst Centre and University of Twente works on industrialization of this process. Both process development as well as equipment solutions are being developed.

From literature it is already known that LIFT can be performed using both thin-film metals as well as silver nanoparticle (NP) inks as donor material [7]. Typically, using thinfilm metal as starting point leads to the smallest deposits. However, thus far, for thin-film metal, no method existed to come to a robust process window that yields small deposits without generating considerable debris. Such debris typically ends up as unwanted contamination that deteriorates device reliability.

The current paper shows an approach to come to a robust, contamination-free process window for thin film metal donor material. Thermal modeling is used in combination with morphology classification of experimentally generated deposits, to identify the optimal process conditions.

Using such clean deposits, continuous metal lines having a width well below $10 \mu \mathrm{m}$ are generated. Thus, we tackled a major roadblock towards the industrial feasibility of LIFT as a full metal direct-write technology that meets the current demands for IC packaging and integration.

The paper first elaborates on the experimental procedure to create deposits which can be classified on morphology. Some typical results as well as the morphology classification are presented. Secondly, modeling approach and results are explained and a match is made with experimental morphology classifications. Finally, conclusions are drawn and future challenges are discussed.

\section{Experimental setup}

Thin film copper was the donor material for all experiments. It was sputtered on standard glass microscope slides with a layer thickness ranging from 200-1200nm. For the experiments a microscope slide holder was used as shown in Figure 2. The holder contains the receiving glass substrate and the donor carrier, with $25 \mu \mathrm{m}$ spacers separating the two slides. A pulsed laser (nano- or picoseond) is fired through the carrier onto the donor layer. In this way, the laser deposits copper onto the receiver as shown in Figure 1. All experiments were conducted in air, at ambient pressure and room temperature.

Before all experiments, the sample holder was positioned such that the laser beam focal point is on the donor layer. The experiments consisted of a series of 'power scans' for each donor layer thickness. In a power scan, multiple lines of pulses are shot at the donor material. The energy per pulse is increased for every new line. Thus, the 'threshold pulse

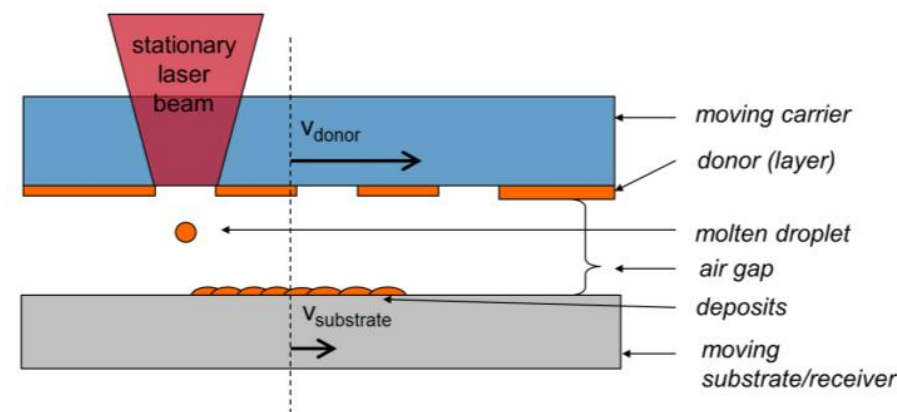

Figure 1: Schematic overview showing the different aspects of the LIFT process. The donor layer is coated onto a transparent carrier, which moves with respect to a stationary laser beam, thus supplying fresh donor material. To make a continuous line from the deposits onto the substrate, the substrate also moves with respect to the laser beam. The laser pulse is absorbed at the carrier-donor interface, where it generates thermal expansion and/or vapor pressure which in turn propels the deposit across the air gap [6]. 
energy' for each donor layer thickness can be determined. The threshold pulse energy is the lowest energy per pulse that results in a deposit. Robust deposits that visually show the least contamination appear just above the threshold energy.

Laser energy can be defined either by total energy content per pulse $(\mu \mathrm{J}$ or $\mathrm{mJ})$, or by fluence which is the pulse energy density in the spot expressed in $\mathrm{J} / \mathrm{cm} 2$. Energy levels can only be compared at the same spot diameter, whereas the use of fluence eliminates this dependency.

Power scans have been made using different types of lasers. Table 1 shows an overview of the main laser parameters. Roughly, two wavelengths and three pulse lengths have been compared.

\section{Experimental results}

For every laser set-up power scans were made. These were analyzed using optical and confocal microscopy. A typical example of such a power scan is shown in Figure 3. It can be seen that the first two energy settings (top left) are contamination free. Increasing the laser energy leads to splashlike features that are either scattered on impact, on the fly, or already during droplet generation. For every laser set-up, a combination of pulse energy and donor thickness was found which resulted in contamination free deposits.

The deposits that result from the power scan were classified according to Table 2. Determination of the deposit type was done visually by a process expert based upon the optical microscopic images. We call a deposit a 'droplet' when only one clear contamination-free droplet is visible; a 'splash' is defined as a main deposit with satellites. When the main deposit starts to disappear and only small satellites are visible, we call it a 'spray'. Another regime, found at higher laser fluence in combination with thicker donor layers is the 'pancake'. This deposit is shaped roughly like a flat disk.

\section{Modeling approach}

To determine the relationship between laser settings, donor layer thickness and deposit morphology, we set up a thermal model. This thermal laser-donor interaction model predicts the state of the material, i.e. the phase at every position in the donor layer, taking into account the latent heat for phase transitions.

We define 5 phases in the donor material: 1: Solid, 2: Melting, 3: Liquid, 4: Boiling and 5: Vapor. We are primarily interested in the phase of the donor material at the interface and at the free surface (Figure 4). In total, there are 15 physical combinations possible since the phase at the free interface always lags behind w.r.t. the phase at the interface. Examples of such combinations are:

- Molten at the interface and solid at the free surface

- Boiling at the interface and melting at the free surface

- Vaporized at the interface and solid at the free surface

In the following, we call these 15 possible phase combinations the 15 regimes (Figure 6b).

We simulated copper as the donor material and soda lime glass as the carrier material. The related material properties are used, together with the linear absorption coefficient of copper at the appropriate laser wavelength. Note that this is a simplification since pulsed lasers exhibit extremely high intensities that are well beyond the linear absorption regime [12]. However, this only has an effect on the absolute value of the laser pulse energy corresponding to a specific phenomenon. Whereas, the qualitative relationship between laser settings and donor phase regime already provides a functional tool for robust process development.

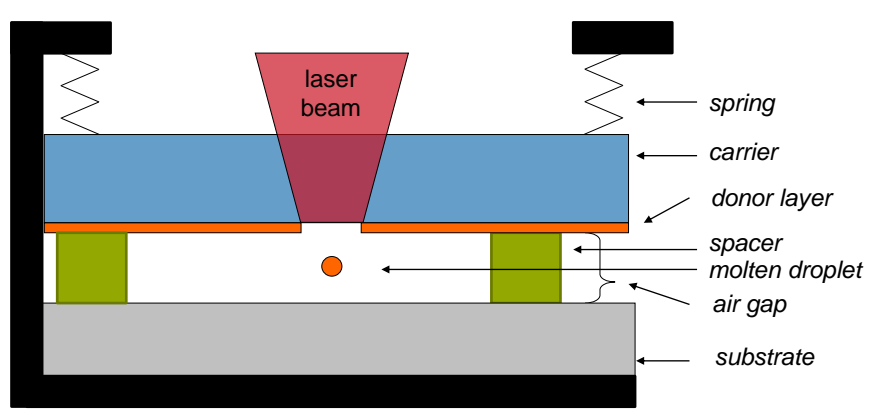

Figure 2: Schematic overview of the droplet generation process that was used for the powerscans. The laser beam is focussed on the donor material where it melts and/or vaporises the donor layer, propelling a deposit. The morphology of the deposit is highly dependent on the fluence of the laser pulse. Therefore, lines of adjenct deposits were written with different fluences for each line. This was done for each combination of donor layer thickness and pulse length.

Table 1: Main parameters of the lasers used

\begin{tabular}{|l|c|c|c|}
\hline $\begin{array}{l}\text { Laser } \\
\text { Type }\end{array}$ & $\begin{array}{c}\text { Pulse } \\
\text { length }\end{array}$ & $\begin{array}{c}\text { Wavelength } \\
{[\mathbf{n m}]}\end{array}$ & $\begin{array}{c}\text { Focus } \\
\text { diameter } \\
{[\boldsymbol{\mu m}]}\end{array}$ \\
\hline Coherent Talisker & $10 \mathrm{ps}$ & $355 / 532$ & 10 \\
\hline IPG YLP G5 & $1 \mathrm{~ns}$ & 532 & 32 \\
\hline Coherent AVIA & $15 \mathrm{~ns}$ & 355 & 17 \\
\hline
\end{tabular}
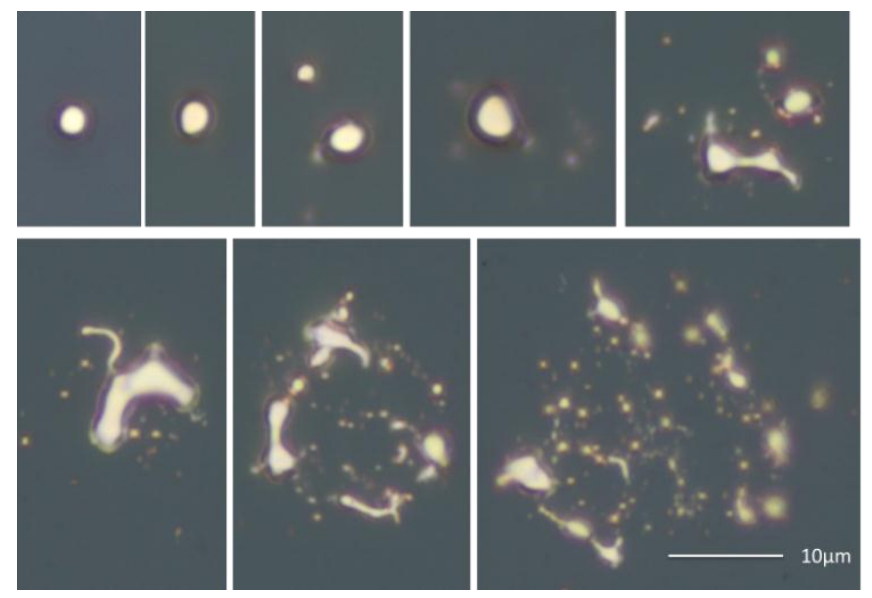

Figure 3: Power scan with Ins laser; 400nm donor layer thickness, increasing energy from left to right, top to bottom. 
Further, in this first approximation, we ignore the influence of the carrier material on the physical process. This means that no optical reflection or absorption is modeled. Also, phase changes of the carrier material are ignored.

The main input parameters that are studied are donor thickness $\delta_{\text {donor }}$, laser pulse energy $E_{\text {pulse }}$, pulse duration $\tau$, laser wavelength $\lambda$ and beam diameter $D$ (radius $R=D / 2$ ). The power of the laser pulse is assumed to be constant over time and we simplify matters by applying a circular laser beam with uniform intensity (flat-top profile). In order to compare the modelling results with the experiments, the energy content of the laser beam in the model (Figure 5 ) is set to the estimated energy absorbed in the experiment, thus ignoring any optical effects of the carrier. The beam diameter $D$ is set to twice the $1 / \mathrm{e}^{2}$-width of the Gaussian beam profile.

Since the total energy under both profiles is kept equal, the fluence $\left[\mathrm{J} / \mathrm{m}^{2}\right]$ of the flat-top profile is lower than the maximum fluence of the Gaussian profile. This means that we calculate a spatially averaged state at the interface and do not incorporate lateral differences.

\section{Description of the model}

The state of the donor material can be predicted at every time step during the laser pulse. At each step a certain amount of energy $E(t)$ is coupled into the donor layer. The energy distribution within the donor layer can be described by a local energy density function $\mathrm{G}\left[\mathrm{J} / \mathrm{m}^{3}\right]$ :

$$
E(t)=\int_{0}^{\delta_{\text {donor }} 2 \pi} \int_{0}^{\infty} \int_{-\infty}^{\infty} G(r, \theta, z, t) r d r d \theta d z .
$$

The function $G(r, \theta, z, t)$ is an intricate function which should incorporate several effects, e.g.:

- Laser energy absorption

- Thermal diffusion in the donor layer

- Heat loss to carrier

- Heat loss to environment at the free surface

- Other losses

The model was set up with the intention to describe the state of the donor material and to find a match with experiments. So we simplified the function $G$, by assuming a perfect thermal contact between donor and carrier, by combining the laser penetration depth with a vertical diffusion length into one exponential term and by neglecting heat loss to the environment at the free surface. We are aware that these assumptions are not $100 \%$ physically correct, but for first order calculations physically acceptable results were expected. With the calculated local energy density function $G$, we can determine the temperature and local state of the material.

Thus, the temperature and state of the material can be predicted at the end of the laser pulse, both at the carrierdonor interface and at the free surface. This can be done for multiple combinations of $\delta_{\text {donor }}, E_{\text {pulse }}, \tau, \lambda, D$ etc. The resulting regimes can be plotted as a function of donor layer thickness and laser energy. This yields regime diagrams that will be shown for different pulse lengths in the next section.
Table 2: Classification of deposit morphologies, identified by optical microscopy and expert analysis.

\begin{tabular}{|c|c|c|}
\hline Deposit morphology & Marker & Name \\
\hline & & $\begin{array}{c}\text { Droplet } \\
\varnothing 3 . .5 \mu \mathrm{m}\end{array}$ \\
\hline & & $\begin{array}{c}\text { Pancake } \\
\varnothing 10 . .16 \mu \mathrm{m}\end{array}$ \\
\hline & & $\begin{array}{c}\text { Splash } \\
\varnothing^{* 1} \sim 12 \mu \mathrm{m}\end{array}$ \\
\hline 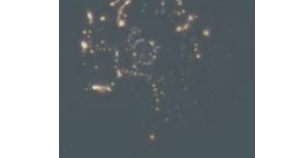 & & $\begin{array}{c}\text { Spray } \\
\varnothing^{* 1} \text { area } \sim 30 \mu \mathrm{m}\end{array}$ \\
\hline
\end{tabular}

*1 The diameter that contains $90 \%$ of the mass of the transferred material

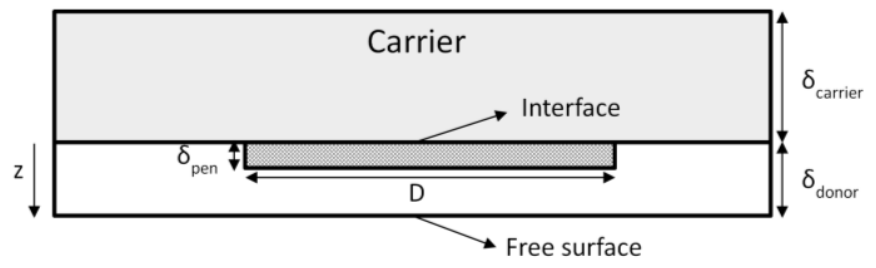

Figure 4: Schematic picture of the donor attached to the carrier. The interface and free surface are denoted.

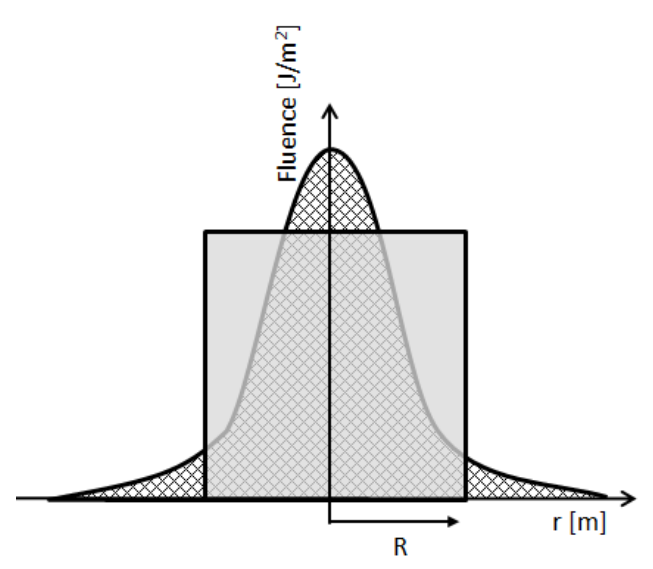

Figure 5: Gaussian profile (experiment) and a flat-top profile (model) with equal energy content. 


\section{Regime diagrams}

Experiments have been performed with three lasers with different pulse lengths and wavelengths (Table 1). Figure 6,7 and 8 show the regime diagrams for the three pulse lengths: 10 ps, $1 \mathrm{~ns}, 15 \mathrm{~ns}$. The regime diagram for picosecond pulses should be interpreted with care, since the time scale related to the energy transfer from electrons the lattice is approximately 7 ps for copper [13]. So for laser pulses in the order of picoseconds or lower, one should take into account electronphonon interactions for a correct calculation of the electron and lattice temperatures. This can be done using the twotemperature model [10], but was beyond the scope of the current study.

In Figures 6,7 and 8, the coloured surfaces correspond to a specific regime, as denoted by the legend. For each regime, the transfer mechanism for deposition is different. For example in regime 9 , where the interface is in a boiling state and the free surface in a molten state, the gas pressure needs to exceed the surface tension (when ignoring adhesion) in order to transfer material. Alternatively, e.g. in regime 7 the interface is in a boiling state and the free surface in a solid state. This means that the gas pressure needs to exceed the critical pressure of the solid layer to initiate transfer of donor material. Experimental morphology classifications have been added to these regime diagrams and are indicated by the black markers (Figure 6). It can be seen that the morphology classification shows a strong correlation with the phase regimes, namely:

- The pancakes are 'only' observed nearby regime 7, where the model predicts that gas production occurs at the interface while the free surface is still in a solid phase.

- The droplets have been observed at energies slightly above the experimental threshold, and lie inside or close to the regimes where the model predicts that the free surface is melting or in a liquid state. Slightly above the threshold, we still observe local, contamination free deposits.

- Increasing the laser pulse energy at a specific donor thickness generally results in larger deposit areas and more contamination. The 'spray' deposit with a lot of contamination can have different causes, depending on the position in the regime diagram. For instance, in regime 7 pancakes with high kinetic energy fragment on impact.

Alternatively, the solid layer might fragment during the initial release. In regime 9, droplets with high velocities can be transferred that splash on impact. There are also other regimes which can lead to a phase explosion at the interface and cause a liquid vapour mixture spray towards the receiver. Even a solid/liquid/vapour mixture is possible

One way to determine the influence of laser parameters on deposit morphology, is by conducting exactly the same experiment at different pulse lengths. The laser pulse length has significant influence on the regime diagram. In our first experiments, we fixed the donor thickness at $200 \mathrm{~nm}$ and tested three different laser pulses: 10ps, $1 \mathrm{~ns}$ and, $15 \mathrm{~ns}$. From the regime diagrams it is clearly visible that these experiments were done in different regimes. For longer pulses, specific regimes shift towards a larger donor thickness. This can be understood by following the position of a specific regime when the laser pulse length is increased. When one fixes the donor thickness, specific regimes can appear or disappear while changing the laser pulse length.

Short pulses and thick donor layers result in phase differences between the interface and the free surface, while long pulses and thin donor layers can result in a more equal state at the interface and at the free surface. These differences in regimes are correlated to the classification of deposit morphologies in Table 2. According to the regime diagram for $\tau=1 \mathrm{~ns}$, we found contamination free deposits near regime 4,5 and 7. In the diagram for $\tau=15 \mathrm{~ns}$, the experiments fall into other regimes and result in deposits with more contamination: ' $*$ ' and ' $x$ ' markers in regime 8,9,10 and 14 in Figures 7 and 8 .

Increasing the laser energy within a specific regime results in deposits with more contamination. Depending on the regime, different causes can be identified. For example in regime 7,8 and 9 the gas production rate near the interface increases when the laser energy is higher, resulting in higher kinetic energies of the material to be transferred. This can eventually result in higher impact velocities and more extensive splashing/fragmentation on impact and thus more contamination. On the other hand, higher deposit velocities and higher temperatures may be beneficial for adhesion and electrical conductivity.

\section{Application}

Applying the droplet regime at the $15 \mathrm{~ns}$ laser, droplets were combined into arrays and lines, to demonstrate the potential for metal patterning. In Figure 9 an array of contamination free droplets is shown. In this array each line was shot with a different laser fluence, but still all droplets are contamination free. This demonstrates the width of the fluence process window that we identified. In Figure 10 a pattern written using LIFT is shown. The pattern shows the capability of the LIFT process to produce continuous lines in a variety of shapes. 


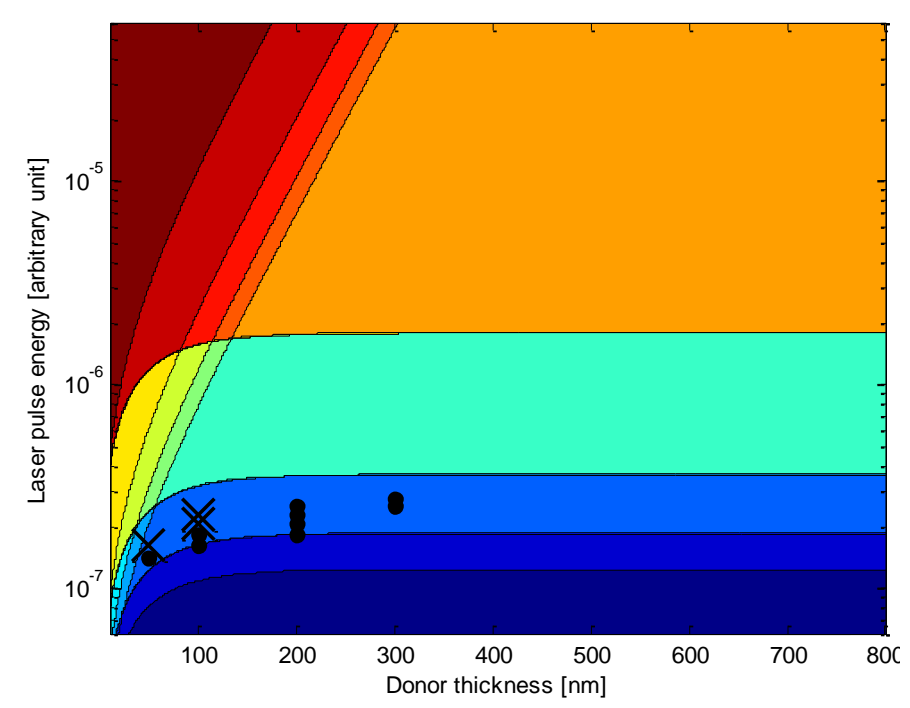

(a)

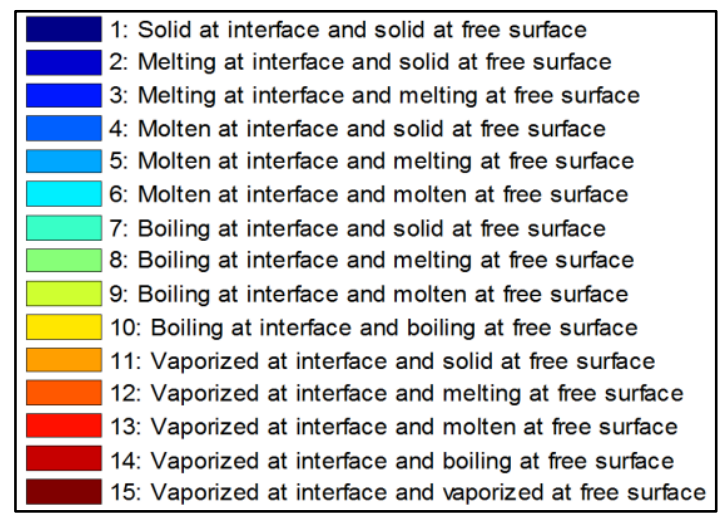

(b)

Figure 6:a) Regime diagram of the donor material as a function of laser fluence (qualitative) and donor layer thickness, for $\tau=10 \mathrm{ps}$ and $R=10 \mu \mathrm{m}$. Experimental deposit morphology is indicated by black markers: $\bullet=$ droplet, $\mathrm{O}=$ 'pancake', $x=$ splash, $*=$ spray. b) Legend of the 15 regimes which are shown as colors in the regime diagrams.

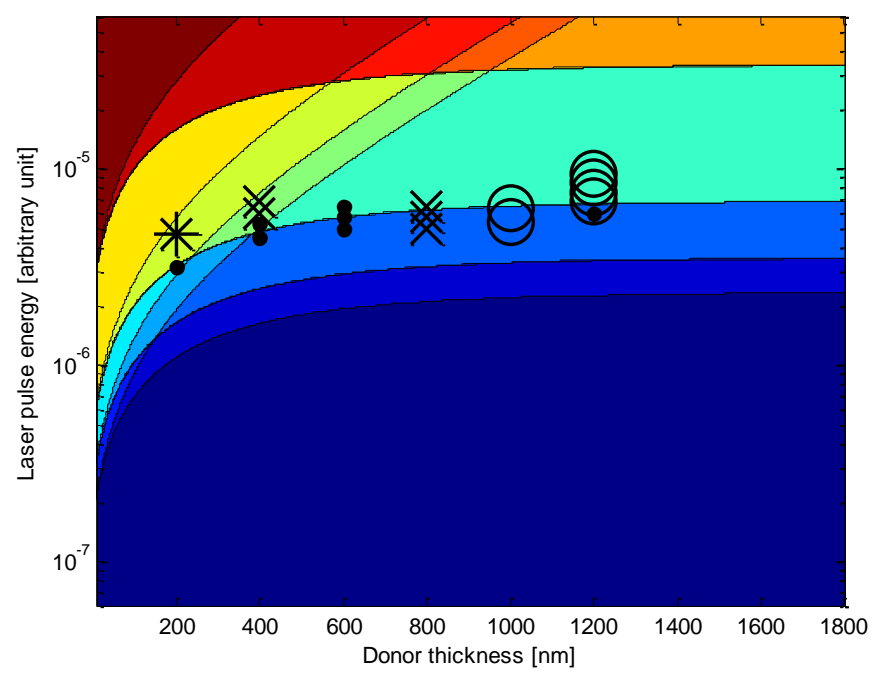

Figure 7: Same as figure 6, but for $\tau=1 \mathrm{~ns}$ and $R=16 \mu \mathrm{m}$.

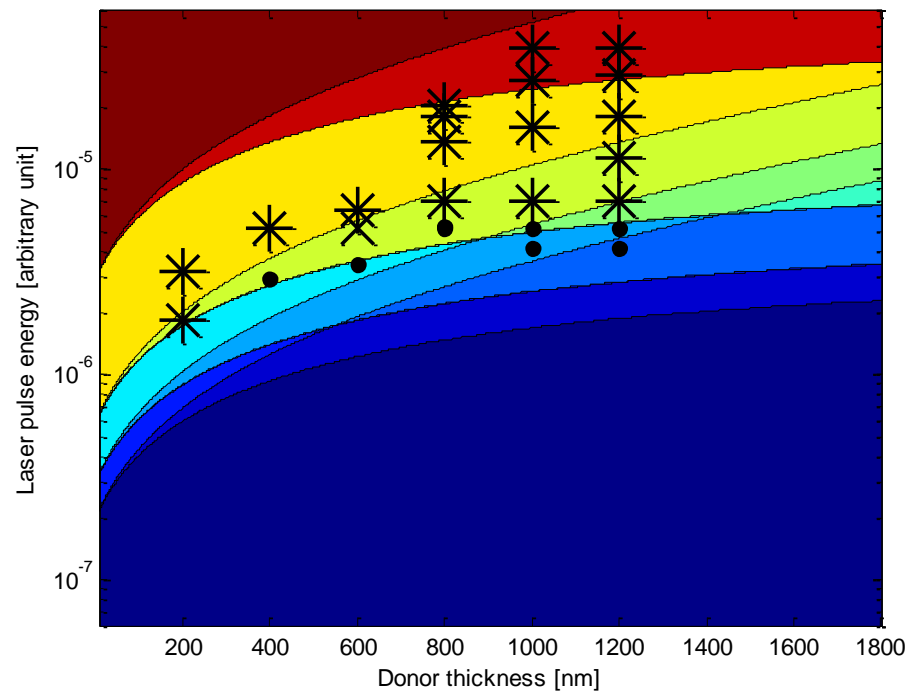

Figure 8:Same as Figure 6 but for $\tau=15 \mathrm{~ns}$ and $R=8.2 \mu \mathrm{m}$.

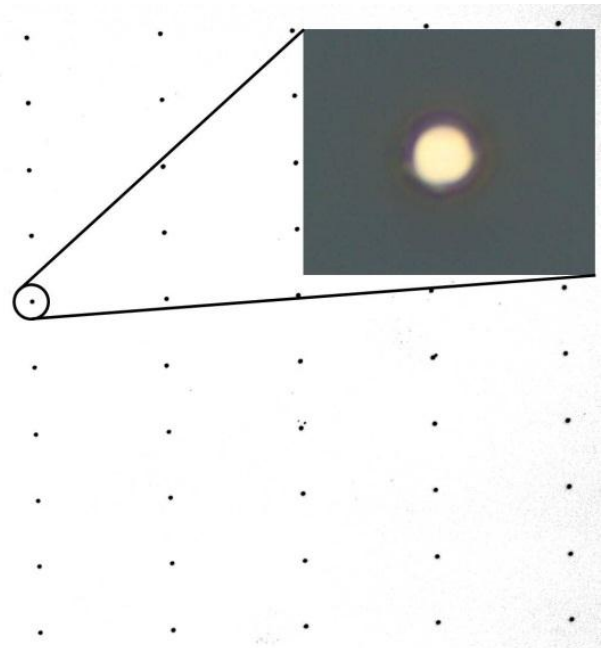

Figure 9: Array of contamination free deposits. The diameter of this droplet is about $5 \mu \mathrm{m}$.

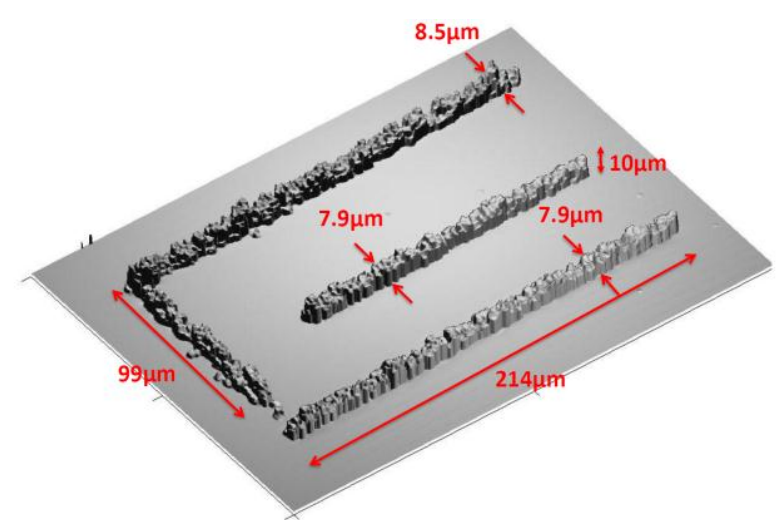

Figure 10: This pattern, deposited using LIFT, shows the capability of the process to deposit copper lines in a variety of shapes. 


\section{Conclusions and discussion}

The present work shows a very strong correlation between deposit morphology and modeling results. This improves the understanding of the relationship between laser settings, donor layer thickness deposit morphology e.g. size, shape, number of deposits, liquid/solid phase distribution, impact behavior etc.

Summarizing, to achieve small and clean deposits, boiling at the interface should be avoided. For pancake structures, a solid free interface at thicker donor layers seems crucial. The splashing regime seems to be small and less well defined. Boiling, or vapor at the free surface always leads to spray deposits.

Using this knowledge, we are now able to choose a laser setting and a donor layer thickness that together lead to a welldefined and reproducible morphology of the deposits, ranging from clean single droplets to a diverging spray. Using this clean droplet morphology, it is possible to create well controlled lines and arrays of metal droplets smaller than $5 \mu \mathrm{m}$.

Being able to determine robust process windows that lead to contamination-free deposits, is a prerequisite for any industrial application. Hence, the current work is a major step towards such industrial use in for instance IC packaging and integration. Furthermore, the ability to choose deposit morphology over a range of shapes increases the field of use even more and emphasises the versatility of this deposition method.

Finally, we show that in future studies of LIFT, one should be careful with the comparison between experimental results, since experiments may actually be executed in different regimes. In this paper, we clearly illustrate the differences between picosecond and nanosecond lasers via the regime diagram and the according consequences for optimal donor thickness.

\section{Future}

The thermal-model that has been developed was intended for a first order qualitative study which can be improved by:

- the implementation of a more realistic beam profile

- using a more sophisticated prediction of the energy distribution in the donor and carrier material

- $\quad$ adding a contact resistance between carrier and donor material

- $\quad$ incorporating the state of the carrier material.

Especially the latter should be studied since Röder [9] suggests that the phase transition of the carrier material can be the main cause for the propelling force in the LIFT process. That would significantly alter the regime diagram.

In the application of the contamination-free droplet regime, the electrical quality of the metal tracks is one of the first things that needs further investigation. Also, the interaction of deposits with the substrate as well as with previous deposits has to be optimized, to achieve reliable tracks with the required (electrical) properties.

Finally, to come to industrial application of the LIFT process, implementation of the process in cost-effective tooling is essential. TNO is currently cooperating with different industrial partners to come to tooling solutions that can deposit metal tracks at sufficiently low Cost-ofOwnership, for different applications, such as: chip interconnects, conductors for solar cells and electrical grids in displays.

\section{References}

1. W. Cui et al, "Gold nanoparticle ink suitable for electricconductive pattern fabrication using in ink-jet printing technology." Colloids and Surfaces A 358 (1-3), 35-41 (2010).

2. J. Hoornstra et al., "Stencil print applications and progress for crystalline silicon solar cells", 24th European Photovoltaic Solar Energy Conference and Exhibition, (2009), Hamburg, Germany.

3. M. O'Reilly et al., "Jetting Your Way to Fine-pitch 3D Interconnects", Chip Scale Review 14(5) 18-21 (2010) .

4. J.Chung et. Al., 1259-1261, "In-tandem deposition and sintering of printed gold nanoparticle inks induced by continuous Gaussian laser irradiation." Appl.Phys. A 79, 1259 - 1263 (2004).

5 Tummala R. "Fundamentals of Microsystems Packaging", McGraw-Hill (2001) ISBN: 9780071371698.

6 Banks D.P., "Femtosecond Laser Induced Forward Transfer Techniques for the Deposition of Nanoscale, Intact, and Solid-Phase Material, 2008, $\mathrm{PhD}$ thesis University of Southampton.

7 G. Oosterhuis et al., "Laser Induced Forward Transfer of interconnects for 3D integration", ECS Transactions, 41 (43) 81-90 (2012).

8 Narazaki A. et al. 2009, "Nano- and microdot array formation by laser-induced dot transfer", Applied. Surface Science 255 (2009) 9703-9706.

9. Röder, T.C., "Physical Model For The Laser Induced Forward Transfer", Applied Physics Letters, 100 (7) (2012).

10 Chen, J.K. et al, "Modeling of femtosecond laser-induced non-equilibrium deformation in metal films", International Journal of Solids and Structures 39 (2002), pp. 31993216.

11 Momma, C. et al, "Precise laser ablation with ultrashort pulses", Applied Surface Science, 109/110, (1997), pp. 15- 19 .

12 Eason, R., Gamaly, E.G. et al, "Pulsed Laser Deposition of Thin Films: Applications-Led Growth of Functional Materials: Chapter 5:Ultrafast Laser Ablation and Film Deposition", 2007, John Wiley \& Sons, Inc.

13 Gamaly, E.G. et al, "Ablation of metals with picosecond laser pulses: Evidence of long-lived non-equilibrium surface states", Laser and Particle Beams (2005), 23, pp. $167-176$. 